\title{
Development and Embodiment of Automatic Location Tracing Service for Rescue Requester
}

\author{
Sun-Hyo Kim ${ }^{1}$, Jung-Hun Kim ${ }^{1}$, Hee-Sun $\mathrm{Kim}^{2}$, Sung-wook Yoon ${ }^{*}$
}

\begin{abstract}
This study developed a design to estimate the location of rescue demander using the sound system at disaster site, in the conditions of indoor positioning cannot be performed properly. It is a location tracing system through smart phone application. It generates sound wave information of rescuer at the rescue site, and it can be used as assistant means for indoor location tracing at the disaster site using sound wave receiving measurement technology.
\end{abstract}

Key Words: Location Based Service, Disaster Monitoring and Transmitting System, Disaster Information, Sonic Triangulation Tracking.

\section{INTRODUCTION}

Large-scale disasters rarely happen, but, if such a disaster happens, it causes immense casualties. In such a case, the main cause of casualties is inefficient rescue efforts[1]. Thus, it is necessary to develop global positioning system for rescue efforts.

The biggest cause of inefficient rescue efforts is difficulty of positioning the rescue demander. In existing rescue efforts, rescue workers try to locate those who ask rescue using their visual and hearing senses. Specific conditions in disaster sites such as smoke, debris, dust, and noise, etc. hamper finding out the rescue demanders. Such conditions force rescue workers to spend golden time, resulting in heavy casualties [2].

In addition, the current rescue efforts have some problems in emergency information, site information (building structure map, topographical map, and surrounding environment, etc.) the real-time communication system to cooperate with related organizations. And, it is difficult to apply collected information while doing rescue works.

In particular, with personalized devices being commonly used, it has become easier to use sensor-based positioning of smart phone, rather than using existing specific devices. This study is to develop a system of estimating the location of the object of rescue and indicating it on the dashboard by using ultrasonic waves beyond the $16 \mathrm{KHz}$ range defined commonly as ultrasonic wave range.

\section{RELATED RESEARCHES}

The size of the global positioning service market is 24 billion dollars in 2017, and is increasing annually by $39.77 \%$. It is predicted that, by 2021 , the market will grow up to 96 billion dollars [3].

With the development of Internet of Things, Cloud Computing, Big Data, Mobile, etc. penetration rate of smart phone has increased, and cloud service, IoT system, and big data have become available. And, with acute competition and expanded investment on positioning technology among firms led to rapid development of positioning service indoors and outdoors (Fig. 1).

Location Based Service (LBS) provides user with location information using information on user's location and landmarks in the neighborhood. The user's handset incessantly receives signals from mobile communication networks and global positing system (GPS) satellite

\footnotetext{
Manuscript received Dec 5, 2020; Accepted Dec 28, 2020. (ID No. JMIS-20M-12-039)

Corresponding Author (*): Sung-wook Yoon, Dep. of Multimedia Engineering, Andong University, uvgotmail@nate.com

${ }^{1}$ Buildup Co, Ltd. Andong, South Korea. buildup8020@hanmail.net

${ }^{2}$ Andong National University, Andong, South Korea, hskim@anu.ac.kr
} 
signals, and the user, using the received information, can figure out his or her position. And, using the location information, the LBS creates new services.

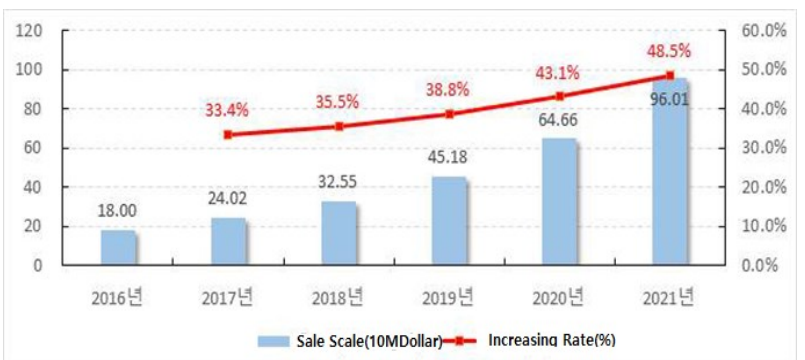

Fig. 1. Location tracking service market sales volume trend (2016-2021).

In 2019, the sales of the LBS industry were 1 trillion and 591.8 billion won, and are expected to grow 250 billion won to 1 trillion and 841.8 billion won. With the increase of penetration rate of smart phone and that of the demand of LBS in Korea, various LBS-used services and applications are being released. By combining with big data analysis, the development of such technologies and corresponding sales are expected to increase further [4], [5].

The increase of LBS use in all socio-economic activities of individuals, forms, a and the government works as the driving force of the growth of the LBS market. In particular, the government and the public sector are increasingly using LBS. The sales of LBS in the safety and security area were 64.1 billion won, and increased in 2018 by $29.5 \%$ to 83.0 billion won. It is expected that the size of the sales in this area will continue to increase.

The penetration rate of smart phone in Korea is $95 \%$, No. 1 in the world. Most of them are equipped with GPS, Wi-Fi, and Bluetooth Low Energy (BLE). The competition among firms to develop the positioning technology by mixing them is being accelerated. Currently, GPS technology used for outdoor positioning is commonly used now, and the technology for indoor positioning is being actively developed [6]. Never Labs developed the indoor AR navigation system by combining various sensor data and visual localization technology.

iBeacon is the device which provide location information from the signals like figure, light, sound, or electric wave. Using iBeacon, Bluetooth 4.0 was adopted in 2010, and With the release of BLE technology providing low electricity and unlimited time synchronization between terminals, mobile payment firms like Apple and PayPal, etc. release BLE-style beacon products.
Wi-Fi Fingerprint technology was developed by changing the intensity of the received Wi-Fi signal into database, and measuring the location of user using the intensity. It is the technology to measure indoor position of the user by modeling Wi-Fi signals which change in non-linear forms depending on time and location as NonGaussian. It is most actively used.

With activation of distribution of smart devices in Korea in 2010, application for LBS technology-based patents which can be applicable to smart devices has become more active. In the situation of intellectual property rights (including patent) on related technology/products, patents in America take $42 \%$ of all the patents on positioning technology in the world [7]. They are highly evaluated, and the American technology is growing rapidly.

In 2011, Telecommunications Technology Association (TTA) started to standardize devices developed by Korean firms, and, in 2014, TTA established the TTAS. In 2018, TTA PG904 is promoting domestic standardization of positioning and navigation servicerelated technology. Expecting the great changes of telecommunication environment into $5 \mathrm{G}$, positioning standardization activities in vehicular telecommunications environment are going on actively led by 3 GPP and OMA[8], etc. (in Table 1).

Table 1. Current status of standards related to LBS in Korea(TTA Korea Information and Communication Technology Association).

\begin{tabular}{|c|c|c|}
\hline St. No & St. Name & Estab. \\
\hline & Disaster Victim Location & \\
\hline $\begin{array}{l}\text { 1 IAK.KU- } \\
06.0504\end{array}$ & $\begin{array}{l}\text { Analysis System Stage 2: } \\
\text { Structure }\end{array}$ & $\begin{array}{l}2019- \\
12-11\end{array}$ \\
\hline TTAK.KO- & $\begin{array}{l}\text { [Revision] Indoor location-based } \\
\text { service for personal and vehicle }\end{array}$ & 2019- \\
\hline part4/R2 & $\begin{array}{l}\text { terminals-Part } 4: \quad \text { Service } \\
\text { interface }\end{array}$ & $12-11$ \\
\hline $\begin{array}{l}\text { TTAK.KO- } \\
06.0486\end{array}$ & $\begin{array}{l}\text { Emergency rescue request } \\
\text { smartphone user interface }\end{array}$ & $\begin{array}{l}2019- \\
06-18\end{array}$ \\
\hline $\begin{array}{l}\text { TTAK.KO- } \\
06.0477\end{array}$ & $\begin{array}{l}\text { Positioning and navigation } \\
\text { performance index for location- } \\
\text { based service and its test method }\end{array}$ & $\begin{array}{l}2018- \\
12-19\end{array}$ \\
\hline $\begin{array}{l}\text { TTAK.KO- } \\
06.0478\end{array}$ & $\begin{array}{l}\text { Fingerprint Map Exchange } \\
\text { Standard for Indoor Positioning } \\
\text { Support }\end{array}$ & $\begin{array}{l}2018- \\
12-19\end{array}$ \\
\hline
\end{tabular}

Technology standardization organizations like 3GPP, 3GPP2, IEEE and others including Korean TTA, and service standardization organizations like OMA, OGC, WiMAX Forum and others are active. TC211, the department of international standards of Geographic Information System under ISO, is doing projects like ISO 19132, ISO 19133, and ISO 19134. with 
'DoubleBlending' strategy of close cooperation, OGC and TC211 under ISO are making synergy effects in international standardization.

\section{DESIGN \& IMPLEMENTATION}

\subsection{Interface Design}

This study designed a system to estimate the location of one who asks rescue in disaster with the positioning method using sound system in the situation where indoor positioning cannot be applied in various ways.

By generating ultra-sound wave over $16 \mathrm{KHz}$ at disaster site, rescue worker can detect specific frequency emitting from the smart device of the one who asks rescue, and figure out the delivery speed and distance through time lag between sending and receiving signals.

Through discernment of smart phones of the user and rescue worker and triangulation using ultra-sound of the central control center, this study made it possible to find out the location of rescue demander through the design where indoor locations of rescuer and rescue worker are received by application of rescuer.

When the disaster letter on the one who needs rescue is received, the application is activated. After GPS is activated, when disaster SMS letter is received, rescue worker executes pop-up of demand of rescue or not through text reader.

As the GPS function of ISO-based application is off at normal times in Figure 2, the user can execute pop-up on rescue demand by pushing the app.

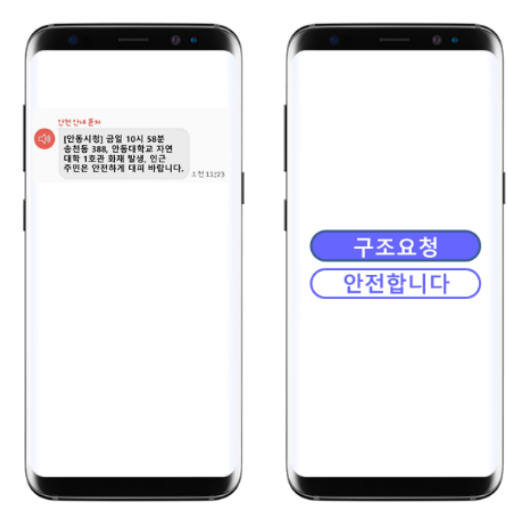

Fig. 2. Disaster application that responds to Korean disaster texts.

If user demands rescue, the GPS function is activated, and position information is sent. If a disaster occurs, apps of Android and IOS two versions allows the one who wants to be rescued to send location information on the standard of the rescue-asking protocol. When background is working, the electricity to send real-time location information is reduced.

\subsection{Basic Concept}

Apps of Android/ISO sensed ultrasound of smart phone, and processed by sending the data to the control center. This study created coordinates by calculating the distance through triangulation through Python 3.x version. This study estimates real-time position on 2-dimensional plane, and inputting 3 coordinates of 3 speakers, and calculates the distance between the one who asks rescue and speakers.

* Moving object $(\mathrm{M})$ current coordinate $=(\mathrm{X}, \mathrm{Y})$

* Speaker $1=$ Coordinate $\left(\mathrm{X}_{1}, \mathrm{Y}_{1}\right)$

* Speaker 2 = coordinates $\left(\mathrm{X}_{2}, \mathrm{Y}_{2}\right)$

* Speaker $3=$ Coordinates $\left(\mathrm{X}_{3}, \mathrm{Y}_{3}\right)$

* Distance to the reference point $\mathrm{d}_{1} / \mathrm{d}_{2} / \mathrm{d}_{3}$

The distance from each reference point to $M$ is calculated $\mathrm{d}$ by Pythagorean theorem. The distance between each standard point and $\mathrm{M}$ is calculated by Pythagorean theorem. By triangulation using RSSI using each $d$ value, this study estimates position. The location of rescue demander is acquired by indicating the common area as the range and getting intersection of sets by triangulation(fig. 3).

$$
\begin{array}{r}
L=20 \log _{10}\left(\frac{4 \pi l}{\lambda}\right)[d B] \\
d=\frac{\lambda}{4 \pi} \cdot 10^{\frac{L}{20}}=\frac{c}{4 \pi f} \cdot 10^{\frac{L}{20}}
\end{array}
$$

* Distance Calculation between two points.

$* \mathrm{c}=$ speed of propagation $/ \boldsymbol{\lambda}=$ frequency

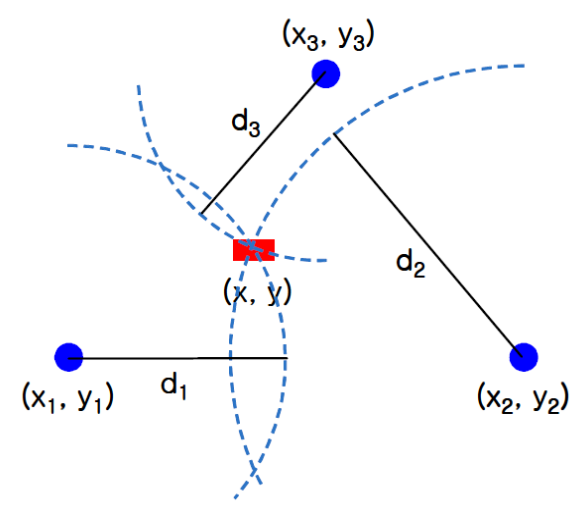

Fig. 3. Adjustment triangulation method. 
Reflection of data and redundant receipt of data caused by reflection of obstacle of ultrasound was filtered with time lag. In calculating RSSI, this study set the ranges of reduction of decibel, and the highest value and the lowest value, and processed them with triangulation.

\subsection{Location measurement using sound waves of the low vocal range}

Location measurement of the low vocal range can be made by checking peak $\mathrm{dB}$ vocal range patterns. Basically, this study set the site drawing of the disaster site or fixed default values of location determination, and input them on the dashboard. Then, it processed $\mathrm{dB}$ and distance calculation through Android-based ultrasound receipt and signal intensity.

When we process signals with audible frequencies in low vocal range, we can process the range by calculating $\mathrm{dB}$ depending on ultrasound receipt through intensity of received signals and getting the average of $\mathrm{dB}$ change range(fig. 4).

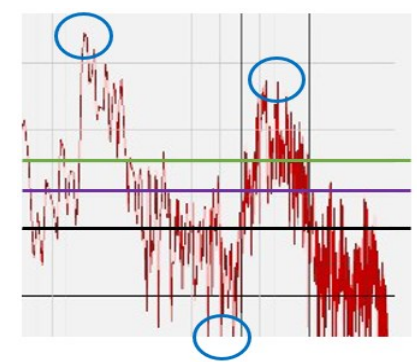

Fig. 4. Processing signal range by calculating sound pressure.

* Calculation of the real-time average of calculated dB the first stage average $\mathrm{dB}$ (upward leveling)

* Based on the first stage average $\mathrm{dB}$, it is possible to get the second stage average $\mathrm{dB}$ of low receipt

* Calculation of average value between the first stage average $\mathrm{dB}$ and the second stage average $\mathrm{dB}$

Distance calculation can be done as follows;

- L (transmit dB-receive dB)

* Transmission dB: $100 \mathrm{~dB}$

- Ultrasound $=20 \mathrm{kHz}$

- C (propagation speed) $=20 \mathrm{kHz}$ propagation speed: 14989.62 (Trim off the 3rd decimal place)

Estimated distance test depending on real distance using $\mathrm{dB}$ of sound waves is as follows.

$$
d=\frac{\lambda}{4 \pi} \cdot 10^{\frac{L}{20}}=\frac{c}{4 \pi f} \cdot 10^{\frac{L}{20}}
$$

- L (transmit dB-receive dB)
Distance calculation through calculating $\mathrm{dB}$ is influenced by noise. Data collection and analysis through Logcat show that the range of error gets bigger depending on the values of distance. It's because of the fact that, even if a specific frequency of sound is sent, the sound wave $\mathrm{dB}$ is affected by surrounding frequencies, and background noises. Figure 5 represents distance testing through sonic processing

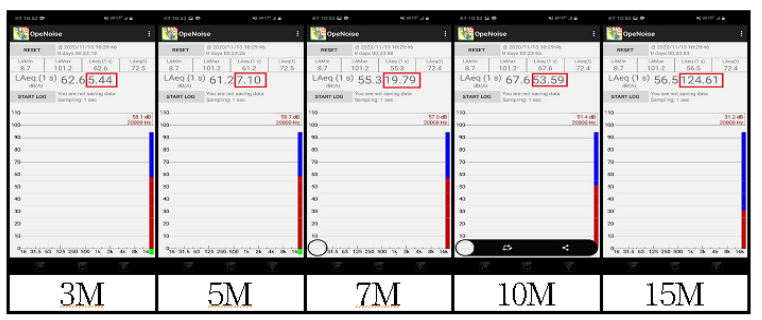

Fig. 5. Distance testing through sonic processing.

\subsection{Application of Location Estimation using Sound Waves of High Vocal Range}

To solve the problems that high frequency is eroded by surrounding low frequencies, and that $\mathrm{dB}$ is reduced to a great extent depending on performance of speaker and HW, this study applied lag time calculation through distance calculation using TDOA to the system.

\subsubsection{Transmitting time (rescuer's app.)}

- Transmitting a specific frequency through rescuer app. connected to speaker

- Storing of transmitted time dB (unit: 1/1000 second)

- Creating the data of frequencies

- Sampling rate: 44,100

- This study sets each frequency and send sound waves, and processed the data by dividing as the number of sample rates in a single block through the number of samples.

* Changing sampling point counts into index values

* Transmission of data time matching each speaker

\subsubsection{Receiving time (rescue demander's app.)}

This study allowed the rescue demander's app. to receive sound waves of specific frequencies from speakers, and process them as algorithm which changes wave forms into spectrum in the FFT Transform method(fig. 6).

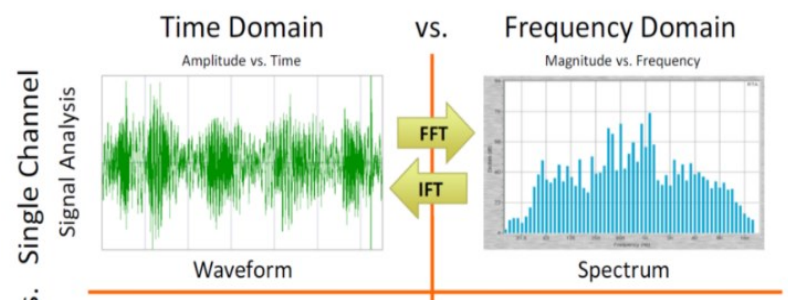

Fig. 6. Spectrum Frequency Domain Process for Waveform [9] 
- Data conversion of received frequency

- Sampling point count index value storage

- Received time dB storage (1/1000 second unit)

- Storing speaker ID

\subsubsection{Control Dash System}

The required time is calculated by subtracting the transmission time from the reception time of the sound wave, and the TDOA method distance is calculated by storing the unique ID of the smartphone

This study made the rescue demander's app. calculate the distances between 3 speakers and the smart phone of rescue demander, and draw three circles using the distances as radiuses of 3 circles, and calculate the median of near distance intersecting points. Figure 7 present sonic triangulation tracking concept.

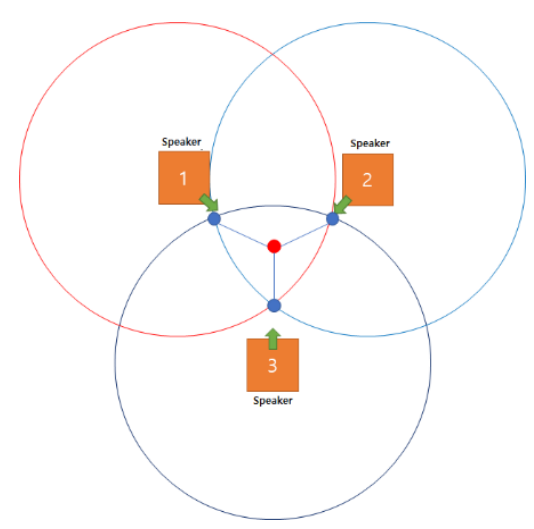

Fig. 7. Sonic triangulation tracking concept.

The time when the sonic data transmission occurs and the data is received is recorded and stored in the database. By resetting the time of transmitting data on transmission device and the time of receiving data on receipt device, demanding the synchronization time with the smallest time difference, and recording the times of transmitted and received data, this study calculated the distance.

Figures 8 and 9 are the UI of the embodied dashboard. The dashboard visualizes the data on differences in received distances, and allows the user to figure out the location of rescue demander, and sends the information to the device of rescuer.

This system embodied the designed system, and measured the app. execution speed with the method to evaluate targeted items. The system perceived the disaster-related message on the device, and visualized the response to execution of app., and figured out the whole link through data acquirement by platform linking of database response speed, and links with different devices. In addition, in triangulation using ultrasound, this study performed function and quantitative target test of the developed device in the gymnasium in Andong University.

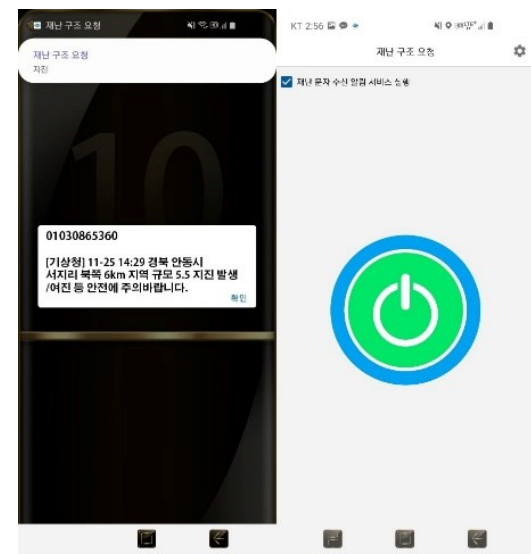

Fig. 8. Disaster application execution in response to Korean.

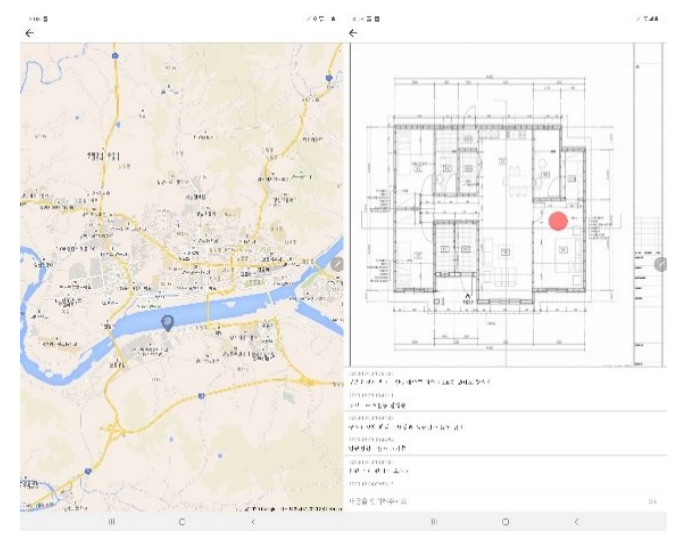

Fig. 9. Location tracking through sound wave database.

The web control system in the form of dashboard can do GIS-based comprehensive modeling, and identify approximate GPS location of rescue demander and that of rescue worker, and process triangulation and positioning of rescue demander through sound wave from near site.

\section{CONCLUSION}

This study developed a design to estimate the location of rescue demander using the sound system at disaster site, in the conditions of indoor positioning cannot be performed properly. It is a location tracing system through smart phone application. It generates sound wave information of rescuer at the rescue site, and it can be used as assistant means for indoor location tracing at the disaster site using sound wave receiving measurement technology.

\section{Acknowledgement}

This paper is the result of a development study planned by SOS Lab of Pohang Techno Park as a 2019 regional 
balanced development SW · ICT convergence technology development (SW service development) with the support of the Ministry of Science and Technology Information and Communication.

\section{REFERENCES}

[1] Ji Won Kim, Yonghee Kim, Melissa Suran, "Emergency-response organization utilization of social media during a disaster: A case study of the 2013 Seoul floods," Journal of Contemporary Eastern Asia, vol. 14, no. 2, pp. 5-15, 2015.

[2] Jong-Tak Park, Chang-Soo Kim, "A study on the development of GIS-based smart disaster prevention system using real time monitoring of city gas leak \& explosion," in Proceedings of International Conference on Information \& Communication Engineering, vol. 8, no. 1, pp. 162-165, 2016.

[3] Technavio(2017), Global LBS Market(2017-2021)

[4] Location Information Support Center, https://lbsc.kr, 2020.

[5] KISA, "Domestic location information industry trend survey," 2019.

[6] Sung-Jun Byun, Yong Han Cho, Sang Keun Choi, Bong Rae Jo, Gun Won Lee, Byung-Hak Min, "Establishment of location-based service(LBS) disaster risk prediction system in deteriorated areas," Korea Academia-Industrial cooperation Society, vol. 21, no. 11, pp. 570-576, 2020.

[7] W. Sakpere, M. Adeyeye Oshin, and N. B. Mlitwa, "A state-of-the-art survey of indoor positioning and navigation systems and technologies," South African Computer Journal, vol. 29, no. 3, pp. 145-197, 2017.

[8] Open Mobile Alliance(OMA), www.openobilealliance.org

[9] FFT Transform, https://blog.naver.com/kon kova /221831156528, 2020.

\section{Authors}

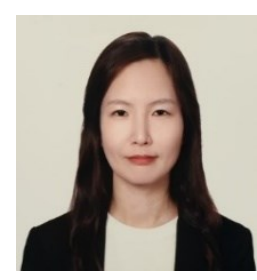

Seon-hyo Kim received her BS in the Department of Multimedia Engineering from Andong National University, Korea, in 2003 and MS degrees in the Department of Computer Engineering from Kyungpook National University, Korea, in 2005.

Her research interests Parallel computation systems for big data analysis and intelligent computation systems for structured and dark data.

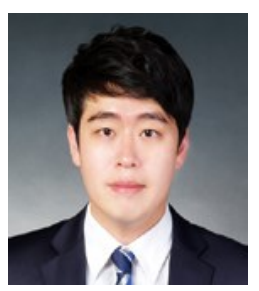

Jung-Hoon Kim received a bachelor's degree from the Department of Bioindustry, Daegu University in 2013.

His research interests include big databased artificial intelligence solutions and data analysis techniques.

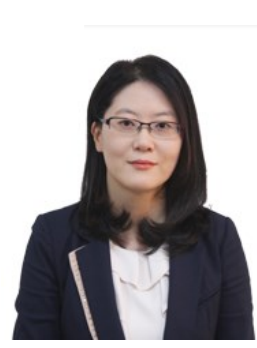

Hee-Sun Kim received her Ph.D degrees in the Department of Computer Science from Kyungpook National University, Korea, in 2001. She is currently professor of Department of Multimedia Engineering in Andong National University, Korea.

Her research interests include mobile applications, HCI and Immersive Media.

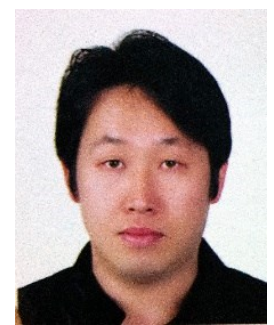

Sung-wook Yoon received his Ph.D degree in the Department of Information \& Communication from Andong University, Korea, in 2016.

He is currently Chief of Buildup Co, Ltd, Korea.

His research interests include Bigdata \& Machin Learning Processing, IoT of Agriculture. 